\title{
Étude de la récupération du collapse par microdensitométrie avec du bois d'Eucalyptus camaldulensis Dehn provenant de Beghla en Algérie
}

\section{Mansour TAzRout ${ }^{1}$}

Mohamed Tahar ABADLIA ${ }^{1}$

Fréderic MотнE

1 Université M’hamed Bougara de Boumerdès

Unité de recherche matériaux, procédés et environnement (Urmpe) Boumerdès

Algérie

2 Inra, Centre de Nancy

Laboratoire d'étude des ressources forêt-bois (Lerfob)

54280 Champenoux

France

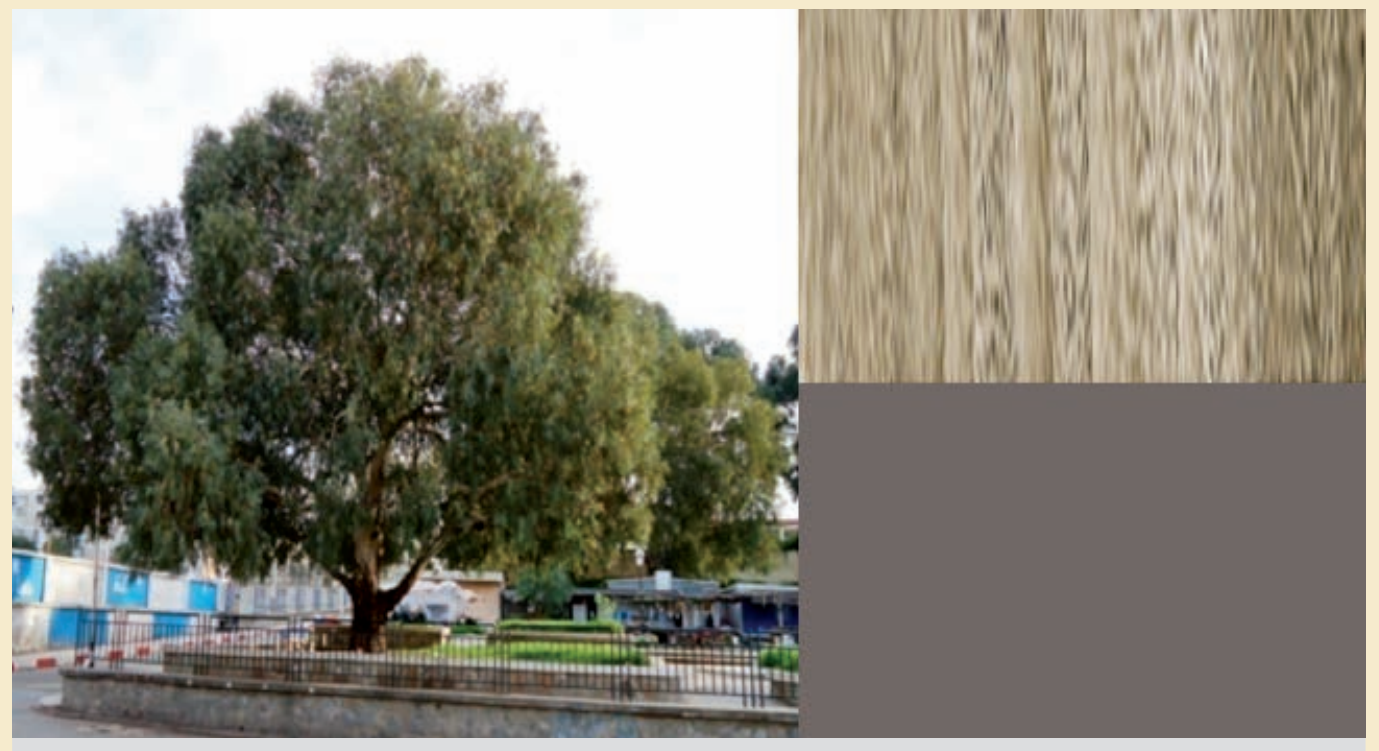

Photos 1.

Arbre Eucalyptus camaldulensis Dehn : ville de Boumerdès. Photos M. Tazrout. 


\section{RÉSUMÉ}

\section{ÉTUDE DE LA RÉCUPÉRATION DU COLLAPSE PAR MICRODENSITOMÉTRIE AVEC DU BOIS D'EUCALYPTUS CAMALDULENSIS DEHN PROVENANT DE BEGHLA EN ALGÉRIE}

L'étude porte sur la récupération du collapse de bois d'Eucalyptus camaldulensis Dehn provenant de la station de Beghla (Algérie), qui occupe une superficie voisine de 500 hectares. Le matériel végétal est constitué de trois arbres dont six barrettes radiales ont été extraites (soit deux barrettes par arbre, représentant le haut et le bas). Une première analyse densitométrique a été effectuée à l'état sec à l'air, puis les éprouvettes ont été reconditionnées, séchées jusqu'à l'humidité initiale, puis soumises à une seconde analyse densitométrique. Le reconditionnement a permis de réduire le collapse dans une large mesure. Les profils obtenus avant et après reconditionnement ont été comparés. La perte de densité relative provoquée par le traitement a été utilisée comme indicateur du collapse. Les résultats montrent que l'indicateur varie en sens inverse de la densité entre éprouvettes et dans le cerne mais décroît sensiblement avec la densité dans l'aubier.

Mots-clés: Eucalyptus camaldulensis Dehn, reconditionnement, récupération du collapse, analyse microdensitométrique.

\section{ABSTRACT}

\section{MICRODENSITOMETRIC STUDY OF RECOVERY FROM COLLAPSE IN EUCALYPTUS CAMALDULENSIS DEHN WOOD FROM BEGHLA IN ALGERIA}

This study deals with recovery from collapse in Eucalyptus camaldulensis Dehn wood from the Beghla station in Algeria, which covers about 500 ha. Three trees were analysed from two radial strips (six strips in all) cut from the top and bottom of each tree stem. An initial microdensitometric analysis was performed on the samples after air-drying. The samples were then reconditioned and dried again to the initial moisture content for a second microdensitometric analysis. Collapse was largely reduced by the reconditioning process The wood density profiles obtained before and after reconditioning were compared, and the relative decrease in wood density on recovery was used as an indicator of collapse. Our results show that the indicator value varied in inverse proportion to the wood density between samples and at the intra-ring level, but decreased substantially with sapwood density.

Keywords: Eucalyptus camaldulensis Dehn, reconditioning, collapse recovery, microdensitometric analysis.

\section{RESUMEN}

\section{ESTUDIO DE LA RECUPERACIÓN DEL COLAPSO POR MICRODENSIMETRÍA EN MADERA DE EUCALYPTUS CAMALDULENSIS DEHN PROCEDENTE DE BEGHLA EN ARGELIA}

El estudio trata de la recuperación del colapso de madera de Eucalyptus camaldulensis Dehn procedente de la estación de Beghla (Argelia) que ocupa una superficie de unas 500 ha. El material vegetal estaba compuesto por 3 árboles de los que se extrajeron 6 listones radiales (es decir, 2 listones por árbol, una de la parte superior y otra de la inferior). Se llevó a cabo un primer análisis densitométrico en muestras secas al aire y, posteriormente, se reacondicionaron y secaron hasta alcanzar la humedad inicial con el fin de someterlas a un segundo análisis densitométrico. El reacondicionamiento permitió reducir el colapso en un grado significativo. Se compararon los perfiles obtenidos antes y después del reacondicionamiento. Los resultados muestran que el indicador varía en sentido inverso a la densidad entre las muestras y en los anillos de crecimiento, pero decrece sensiblemente con la densidad en la albura.

Palabras clave: Eucalyptus camaldulensis Dehn, reacondicionamiento, recuperación del colapso, análisis microdensitométrico. 


\section{Introduction}

Le présent travail s'inscrit dans le cadre d'un projet de valorisation des espèces de bois algériennes du genre Eucalyptus, sous forme de bois d'œuvre, pour une mise à disposition des industriels et constructeurs algériens.

Le choix est guidé par l'importance économique de cette espèce en Algérie : elle représente environ 43235 ha, avec un potentiel de récolte de $144800 \mathrm{~m}^{3}$ /an.

L'Eucalyptus camaldulensis Dehn est utilisable sous forme de brise-vent, de bosquets, d'arbres pour le bétail, pour l'assainissements des marais, le boisement des lits d'oueds, les espaces verts urbains (photos 1 à 6).

Le genre Eucalyptus, myrtacée originaire d'Australie, comprend plus de 700 espèces ou variétés dont le nombre augmente chaque année à la suite d'hybridations tant naturelles qu'artificielles. L'importance économique acquise par ce bois ne cesse de croître chaque année. Cette importance est à attribuer, d'une part, à la pénurie du bois en quantité et en qualité dans certaines régions du monde et, d'autre part, à la plasticité et à la bonne croissance des eucalyptus.

La sensibilité au collapse est l'un des principaux facteurs limitant la valorisation de cette essence. De plus, le bois d'Eucalyptus camaldulensis Dehn est sujet à des attaques d'insectes découverts récemment en Algérie : le phoracantha, Phoracantha semipunctata, et le psylle, Ctenarytaina eucalypti, qui ont ravagé des quantités importantes d'arbres (photo 7).

Dans cette étude, les propriétés du bois sont analysées par microdensitométrie avant et après une opération de reconditionnement visant à réduire les effets du collapse, selon une méthodologie inspirée des travaux préliminaires de Polge (1966) et SeSBou (1981). L'objectif est de vérifier si la perte de densité résultant du reconditionnement peut être un indicateur fiable de la gravité du collapse. Dans l'affirmative, cet indicateur pourrait facilement être mesuré à l'échelle anatomique pour permettre l'étude du déterminisme et de la variabilité de la sensibilité des bois au collapse.

L'analyse a porté ici sur des éprouvettes d'Eucalyptus camaldulensis Dehn provenant de la station de Beghla en Algérie (500 ha environ).

\section{Définition du collapse}

Le collapse est un défaut du séchage qui se traduit par un effondrement de la structure. Il consiste en une contraction considérable et irrégulière du bois, distincte du retrait normalement constaté. Les pièces de bois prennent une forme plus ou moins ondulée (ondulations en surface). Parfois, il y a apparition de fentes internes importantes, invisibles de l'extérieur, qui rendent les bois inaptes à l'emploi. Les parois des cellules sont plissées ou effondrées (figure 1).

Ce phénomène se produit pendant l'évacuation de l'eau libre, alors que le retrait normal apparaît pendant le départ de l'eau liée, en dessous du point de saturation des fibres. Les deux phénomènes se cumulent pendant l'opération de séchage.

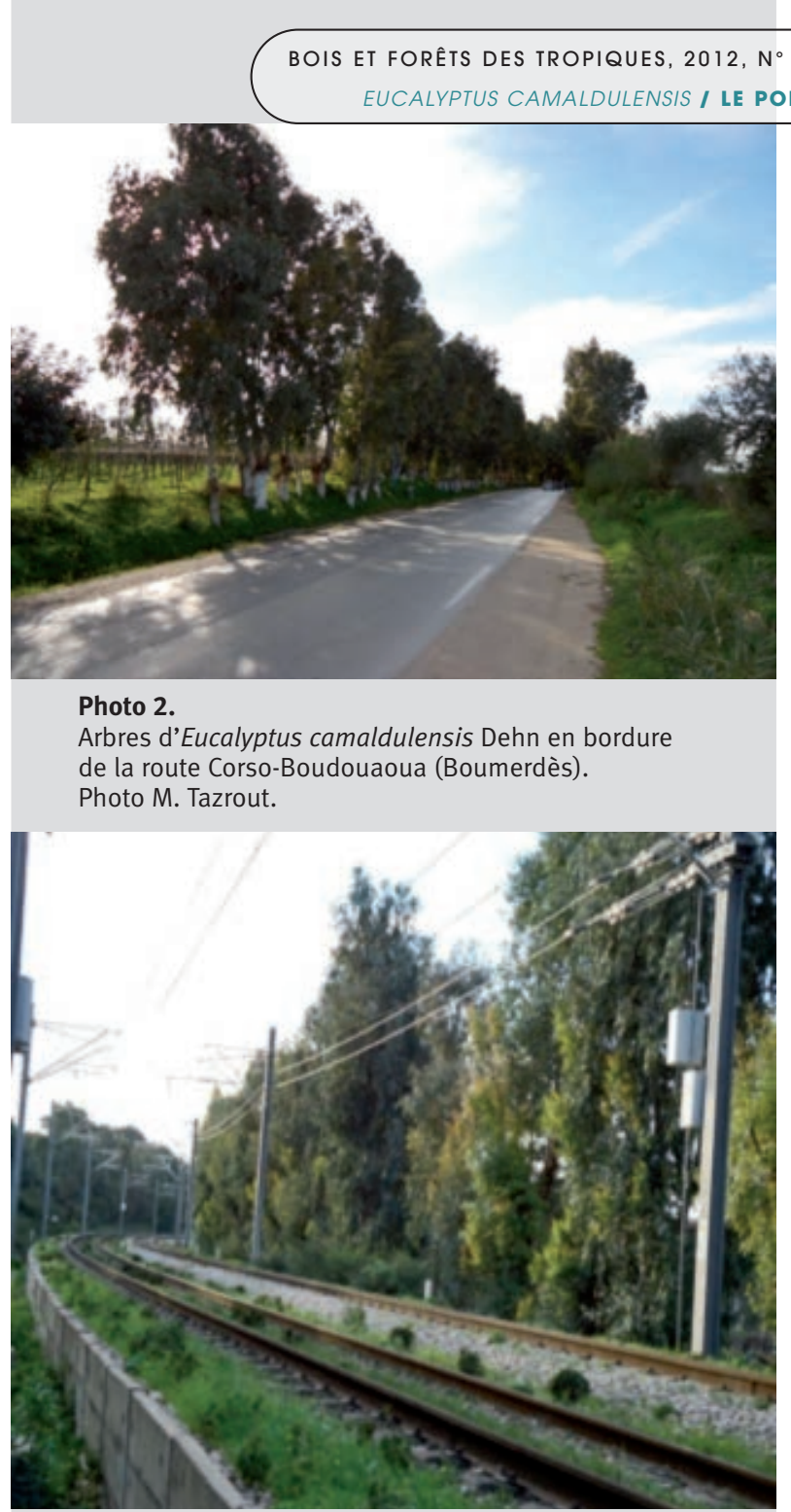

Photo 3.

Arbres d'Eucalyptus camaldulensis Dehn en bordure du chemin de fer Boumerdès-Corso.

Photo M. Tazrout.

À l'heure actuelle, le phénomène de collapse n'est pas complètement élucidé ; il est explicité partiellement par deux phénomènes physiques : la tension capillaire qui s'exerce en cours de séchage dans les cavités des fibres (TIEMANN, 1915 ; HAWLEY, 1931 ; KAUMAN, 1960b ; HART, 1970 ; BARISKA, 1975 ; MACKAY, 1972), et les contraintes exercées par les zones externes, au séchage plus rapide, sur les parties internes à un moment où celles-ci présentent encore une certaine plasticité (CLARKE, 1927 ; STAMM, LOUghborough, 1942 ; TIEMANN, 1941 ; KAUMAN, 1958 ; KAUMAN, 1960a ; RunKEL, 1954 ; SCHNIEWIND, 1960).

Le collapse est le résultat de l'action réciproque entre la répartition de l'eau libre contenue dans le bois et la structure du plan ligneux, d'une part, l'ultrastructure et la résistance des éléments anatomiques, d'autre part (CHAFE, ILIC, 1992a, b et c).

Le collapse peut être en partie maîtrisé par un séchage bien contrôlé. 


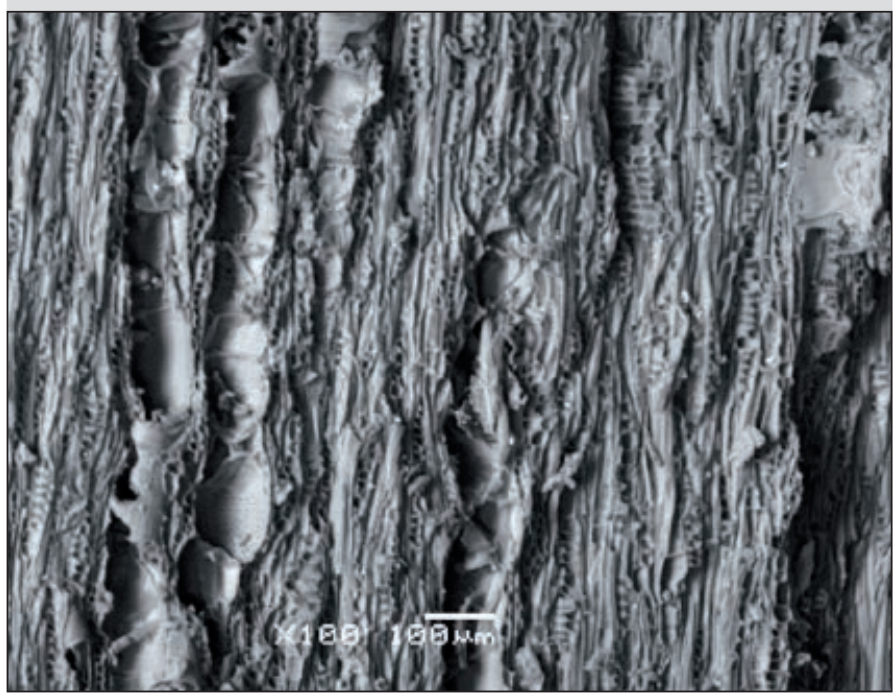

Figure 1.

Coupe tangentielle d'Eucalyptus camaldulensis Dehn collapsé (Algérie).

\section{Variabilité du collapse}

La plupart des bois se collapsent légèrement durant le séchage mais un collapse plus sévère se constate chez certains angiospermes à densité moyenne et chez des bois très humides de quelques gymnospermes.

L'effondrement présente une grande variabilité suivant les genres, les espèces, les arbres et même suivant la position dans l'arbre. Le milieu a aussi une influence.

Une variabilité considérable est souvent constatée entre les arbres d'une même espèce, même s'ils ont poussé dans la même station. Le bois d'arbres jeunes se collapse plus que le bois d'arbres mûrs (CUNNINGHAM, 1827 ; Tiemann, 1913 ; Elliot, 1930 ; PAnkeVicius, 1962). D’autre part, le bois juvénile d'un arbre jeune se collapse plus que le bois juvénile d'un arbre âgé (BISSET, ELLWOOD, 1951).

L'intensité du collapse varie avec la hauteur dans l'arbre; elle est plus importante au pied qu'au sommet (TIEMANN, 1929 ; KAUMAN, 1960a et b ; PANKEVICIUS, 1961). L'effondrement chez beaucoup d'angiospermes se confine généralement dans le bois de cœur mais il existe des cas où le collapse affecte l'aubier (CHUdNOFF, 1953 ; PANKEVICIUS, 1962).

Selon différentes études citées par ILIC (1999), la densité du bois est un indicateur important du collapse chez l'eucalyptus, les bois les moins denses étant plus facilement collapsés. Wu et al. (2006) observent un résultat similaire sur trois espèces d'eucalyptus en distinguant les variations dimensionnelles dues au collapse (liées négativement à la densité) et celles qui sont dues au retrait (liées positivement à la densité).

À l'intérieur d'un cerne, les cellules à parois minces du bois initial ont une faible résistance à la compression et se collapsent généralement beaucoup plus que les fibres à parois plus épaisses du bois final (BISSET, ELLWoOd, 1951). L'analyse microdensitométrique de bois d'eucalyptus reconditionné a permis à ILIC (1999) d'observer que les fissures dues au collapse s'observent préférentiellement dans des

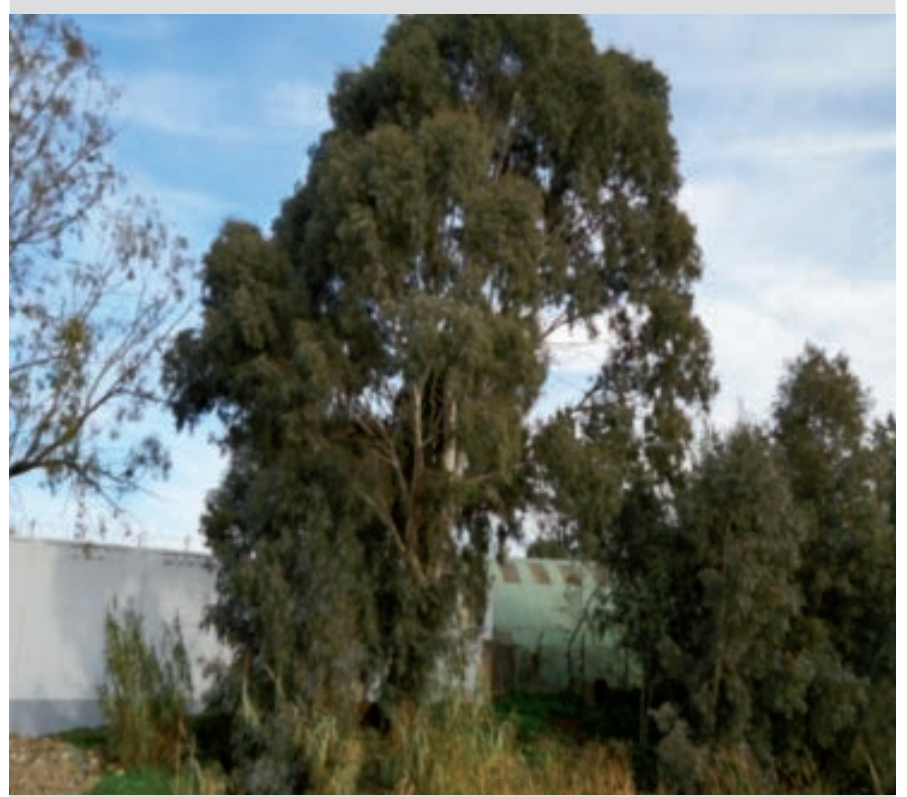

Photo 4.

Arbres d'Eucalyptus camaldulensis Dehn le long de l'oued de Boudouaoua (Boumerdès)

Photo M. Tazrout.

cernes dont le bois initial est anormalement peu dense (moins de $450 \mathrm{~kg} / \mathrm{m}^{3}$ ). Dans des cas isolés, le bois final peut cependant collapser plus que le bois initial selon TIEMANN (1948).

Chudnoff (1961) a observé une diminution de la densité avec la hauteur dans l'aubier mais pas dans le bois de cœur et il attribue cette différence au fait que le dépôt des extractibles dans le bois ne se fait pas de façon uniforme de la base au sommet de l'arbre.

Le taux d'extraits solubles influence le retrait du bois. Il y a en effet une corrélation négative entre le retrait volumétrique et la fraction d'extraits solubles à l'eau (PANKEVICIUS, 1962).

Le collapse est généralement plus important dans le sens tangentiel que dans le sens radial (1,5 à 3 fois). Au cours du séchage à $20^{\circ} \mathrm{C}$, le collapse affecte d'abord les rayons ligneux (KANAGAWA, 1977).

Chafe (1985, 1986), sur 69 espèces d'Eucalyptus, a montré qu'il existe une corrélation négative très significative entre le retrait dû au collapse et l'infradensité du bois. Par contre, il a été observé que la corrélation entre l'infradensité et le retrait volumique total hors collapse est peu ou pas significative pour le genre Eucalyptus (CHAFE, 1986 sur 69 espèces d'Eucalyptus; CHAFE, ILIC 1992a, b et c sur Eucalyptus regnans).

Le retrait initial lié au premier séchage à partir de l'état vert, lorsqu'il est excessif (collapse), n'est pas corrélé à la densité du bois (CHAFE, 1986).

Son ampleur est fonction des conditions hygrothermiques dans lesquelles il est réalisé et de l'histoire de ces conditions.

De plus, il est très possible que le retrait de collapse soit lié à des comportements mécanosorptifs au-dessus du point de saturation des fibres (ARMSTRONG, 1983).

Le retrait hors collapse est une caractéristique spécifique du matériau. En utilisant des éprouvettes de petites dimensions, il est possible d'éviter tout collapse (CHAFE, ILIC, 1992a, b et c). 


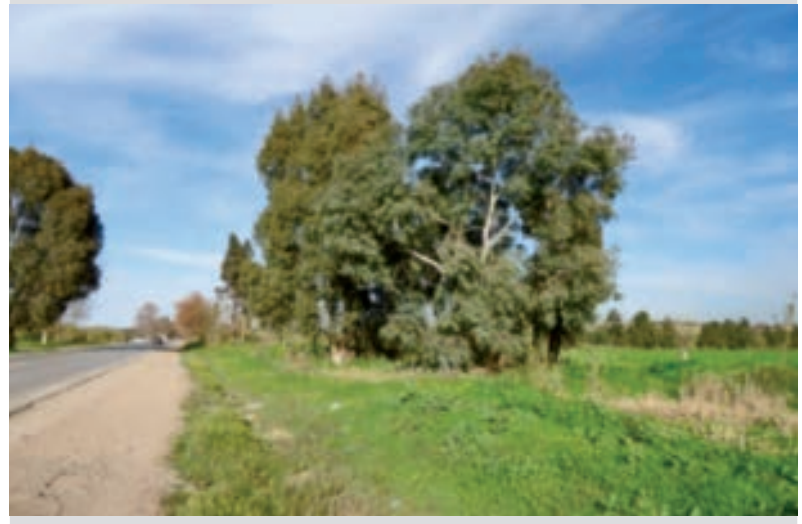

Photo 5.

Arbres d'Eucalyptus camaldulensis Dehn dans les champs de Boudouaoua (Boumerdès).

Photo M. Tazrout.

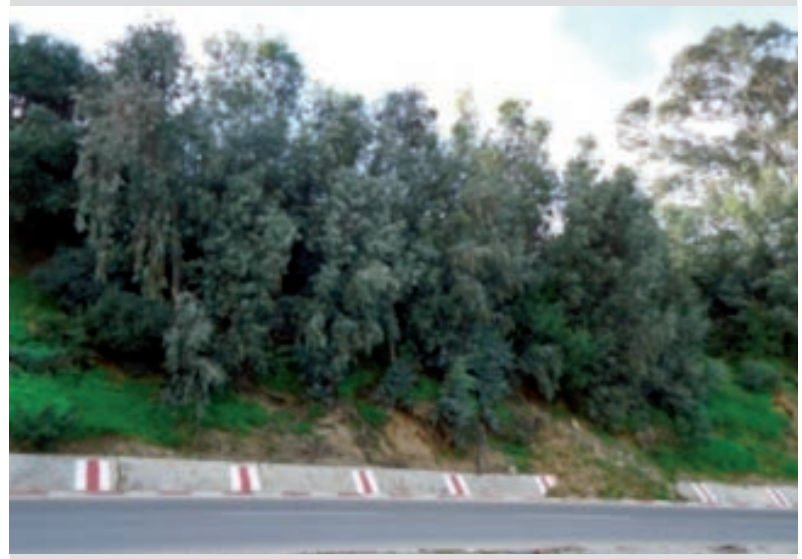

Photo 6.

Peuplement d'arbres d'Eucalyptus camaldulensis Dehn (Boumerdès).

Photo M. Tazrout.

\section{Le reconditionnement}

Une partie des variations dimensionnelles dues au collapse peut être récupérée par un traitement thermique du bois : le reconditionnement. Cette technique consiste à traiter les bois pendant 3 à 6 heures à la vapeur à $100^{\circ} \mathrm{C}$, après un séchage progressif jusqu'à une humidité moyenne voisine de $17 \%$; les dimensions, qui avaient fortement diminué au cours du séchage, augmentent à nouveau pendant cette opération. L'effet du reconditionnement est stable tant que la pièce n'est pas réhumidifiée.

La durée du traitement varie en fonction des dimensions des échantillons : les coupes minces récupèrent instantanément dès qu'elles sont exposées à la vapeur, même à température ambiante, alors que 6 à 12 heures de traitement à $100{ }^{\circ} \mathrm{C}$ sont nécessaires pour des pièces de 5 centimètres $(\mathrm{cm})$ d'épaisseur.

Les meilleurs résultats ont été obtenus avec des températures proches de $100^{\circ} \mathrm{C}$ pendant 3 à 6 heures pour une épaisseur de 2,5 cm (TIEMANN, 1929 ; ELLIOT, 1923, 1930 ; Clarke, 1927 ; Summers, 1929 ; Stilwell, SteVens, 1931).

GREENHILL (1938) montra que l'optimum de récupération est obtenu à une température de $100^{\circ} \mathrm{C}$ quand le bois a atteint 15 à $20 \%$ d'humidité. À des températures supé- rieures, le bois risque de contenir encore de l'eau libre, ce qui peut entraîner une dégradation thermique et la réapparition du collapse après traitement. À des humidités plus basses, le bois n'est pas assez plastique pour récupérer pleinement. En utilisant des températures plus élevées $\left(120\right.$ à $\left.130^{\circ} \mathrm{C}\right)$, des taux de récupération supérieurs sont obtenus (GREENHILL, non publié ; PANKEVICIUS, non publié).

Des températures encore plus élevées $\left(160^{\circ} \mathrm{C}\right.$ pendant 2 heures) ne donnent pas de meilleurs résultats.

Les substances extractibles semblent jouer un rôle positif dans la récupération du collapse (BLAND, 1971).

L'action des deux principaux facteurs du reconditionnement, la chaleur et l'imbibition, n'est pas bien définie. L'action de la chaleur semble la plus importante car il faut élever la température pour que la récupération commence. KAUMAN (in Polge,1966) a montré :

- que l'imbibition varie en sens inverse de la densité du bois ;

- que la récupération, en densité superficielle et en dimensions dans les sens tangentiel et radial, est d'autant plus forte que l'imbibition est plus élevée ;

- mais que des phénomènes d'interaction interviennent dans le bois : le bois de printemps qui est plus collapsé que le bois d'été voyant, par exemple, ses possibilités de récupération limitées par le voisinage de ce dernier.

Le reconditionnement pratiqué actuellement à l'échelle industrielle en Australie est précédé d'un séchage mixte à l'air et au séchoir. Cette technique est satisfaisante tant au point de vue technique qu'économique pour les bois durs difficiles à sécher.

Dans leur aire d'origine en Australie, les bois d'eucalyptus des États de l'Est ont fait l'objet d'un récent rapport élaboré à l'intention de Forest \& Wood Products Australia (Fwpa) par Blakemore et NoRTHWAY (2009). Ce rapport présente un bilan des travaux de recherche sur le problème du collapse du bois, effectués à ce jour par les chercheurs à l'échelle mondiale. Des recommandations pratiques sont données aux industriels sur le séchage et le reconditionnement des bois pour réduire le collapse. Ce rapport servira de guide pour l'élaboration d'une stratégie future par le Fwpa, en vue de valoriser les espèces de bois d'eucalyptus commerciales dans les États de l'Est de l'Australie.

\section{La microdensitométrie}

La radiographie d'une éprouvette de bois permet de visualiser les variations locales de densité. L'analyse microdensitométrique permet ensuite de quantifier ces variations, en particulier pour évaluer l'aptitude du bois à la mise en œuvre (PoLGE, 1966). Les données se présentent sous forme d'un profil radial de densité obtenu par l'irradiation dans la direction axiale ou tangentielle d'éprouvettes de fine épaisseur ( $2 \mathrm{~mm}$ ). Ce profil est ensuite divisé de façon à isoler chaque accroissement annuel que l'on caractérise par un certain nombre de critères synthétiques et conventionnels, tels que la moyenne, le minimum et le maximum de densité (Mотнe et al., 1998). 


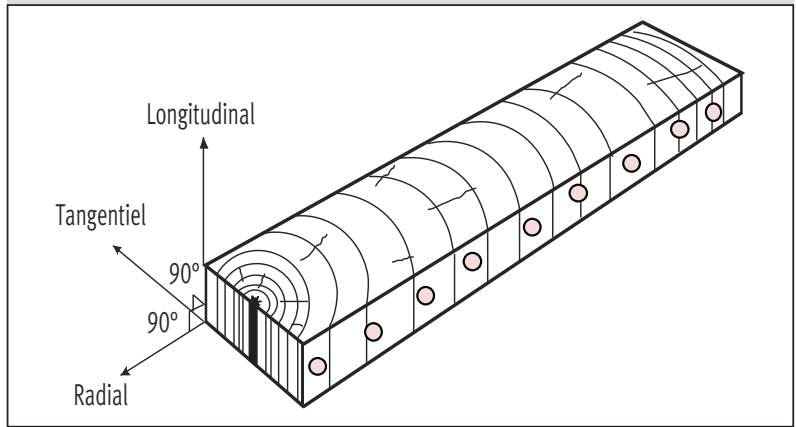

\section{Matériel et méthodes}

Trois arbres âgés de 35 ans et d'une hauteur d'environ 30 mètres $(\mathrm{m})$ ont été prélevés dans la station de Beghla, Algérie (tableau I). Un barreau radial de $35 \times 35 \mathrm{~mm}$ a été découpé à 2 et 6,5 $\mathrm{m}$ de hauteur dans chaque arbre. Les éprouvettes provenant du bas des arbres $a, b$ et c sont notées a1, b1 et c1, celles provenant du haut sont notées a4, b4 et c4.

\section{Préparation des éprouvettes pour la microdensitométrie}

Deux barrettes jumelles de $2 \mathrm{~mm}$ d'épaisseur ont été débitées dans chaque barreau radial (figure 2). Cette opération est réalisée à l'aide de scies fraises jumelées (Thiercelin, Perrin, 1972) de façon à garantir une épaisseur constante de $2 \mathrm{~mm}$ (à quelques centièmes de $\mathrm{mm}$ près) et une orientation correcte dans le plan orthotropique.

Des trous de $0,5 \mathrm{~mm}$ de diamètre alignés radialement et espacés de $5 \mathrm{~mm}$ environ ont été percés longitudinalement sur chaque barrette afin de servir de points de correspondance entre les mesures effectuées avant et après reconditionnement des éprouvettes.

\section{Radiographie des éprouvettes collapsées}

Les éprouvettes ont été mises à sécher "sous contrainte » dans une étuve hygrométrique mise à notre disposition par le Lermab ${ }^{1}$ avec les conditions de séchage suivantes :

- température sèche, $30^{\circ} \mathrm{C}$;

- température humide, $26^{\circ} \mathrm{C}$;

- humidité d'équilibre, $14 \%$.

Après pesée à l'état sec à l'air, les éprouvettes ont été posées sur un film radiographique et irradiées pendant 4 heures (tube à rayons $X$ placé à $2,5 \mathrm{~m}$ du film, avec une tension accélératrice de 7,5 kilovolts et une intensité de 12 milliampères). Une cale comprenant trois paliers d'épaisseur homogène et une rampe d'épaisseur linéairement croissante, usinée dans un matériau polymère de densité connue (kémétal), a été radiographiée en même temps que les échantillons. Les clichés ont ensuite été développés et numérisés à l'aide d'un scanner avec une résolution de 1000 points par pouce. Les niveaux de gris ont ensuite été convertis en densité du bois en tenant compte de l'épaisseur de chaque éprouvette par régression polynomiale sur les valeurs connues de la cale en kémétal à l'aide du programme CRad, développé au Lerfob².
Figure 2.

Orientation d'une barrette de $2 \mathrm{~mm}$ d'épaisseur avec la rangée de trous permettant la correspondance avant et après reconditionnement.

\section{Reconditionnement des éprouvettes et radiographie après reconditionnement}

Le reconditionnement a été effectué sous autoclave dans de l'eau portée à environ $120^{\circ} \mathrm{C}$ pendant une durée de 15 minutes.

Les éprouvettes ont ensuite été remises à sécher sous contrainte jusqu'à l'obtention d'un poids aussi proche que possible de l'état sec à l'air initial, puis à nouveau radiographiées dans les mêmes conditions que précédemment. La comparaison des poids mesurés avant et après reconditionnement (en excluant les éprouvettes a1 et a4 desquelles des fragments se sont détachés pendant l'opération) montre cependant que l'humidité du bois était plus élevée de $0,5 \%$ lors de la seconde radiographie.

\section{Traitement des images radiographiques}

Les éprouvettes n'ont pas la même forme avant et après reconditionnement et ne sont pas orientées de la même façon sur les radios.

Les trous percés sur chaque éprouvette, qui apparaissent comme des taches sombres sur les images radiographiques, ont été utilisés pour mettre en correspondance les mesures avant et après reconditionnement malgré les déformations radiales : le programme Cerd, développé au Lerfob, a été utilisé pour délimiter des fenêtres de mesure rectangulaires, larges de $2 \mathrm{~mm}$ dans la direction tangentielle et commençant $2 \mathrm{~mm}$ au-dessus de chaque trou (segments verts sur la figure 3 ).

\footnotetext{
${ }^{1}$ Laboratoire d'études et de recherche sur le matériau bois (Unité mixte de recherche AgroParisTech Inra-Université Nancy I).

2 Laboratoire d'étude des ressources forêt-bois de l'Inra (Institut national de la recherche agronomique, France, Centre de Nancy).
}

Tableau I.

Description des arbres échantillonnés d’Eucalyptus camaldulensis Dehn provenant de Beghla, Algérie).

Arbre

Âge

Circonférence à $1,30 \mathrm{~m}$

Hauteur totale

Position de l'arbre dans le peuplement

Inclinaison globale du tronc (1)
35 ans

$130 \mathrm{~cm}$

Env. $30 \mathrm{~m}$

Bordure piste

$10 \mathrm{~cm}$ b

35 ans

$122 \mathrm{~cm}$

Env. $30 \mathrm{~m}$

Bordure piste

$13 \mathrm{~cm}$
35 ans

$113 \mathrm{~cm}$

Env. $30 \mathrm{~m}$

Montée

$0 \mathrm{~cm}$

(1) Mesure de l'écart à la verticalité du tronc par fil à plomb sur une hauteur de 2,10 m. 
a1

W.

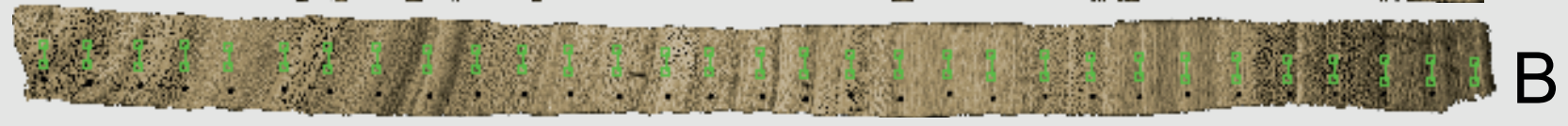

a4

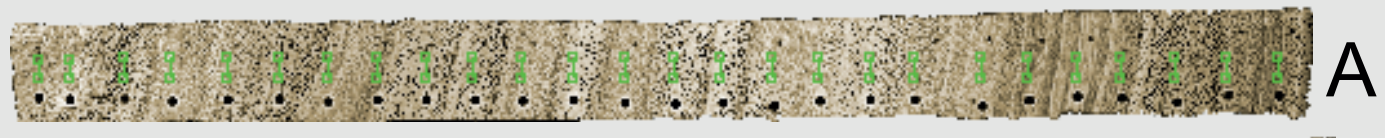

1.4.
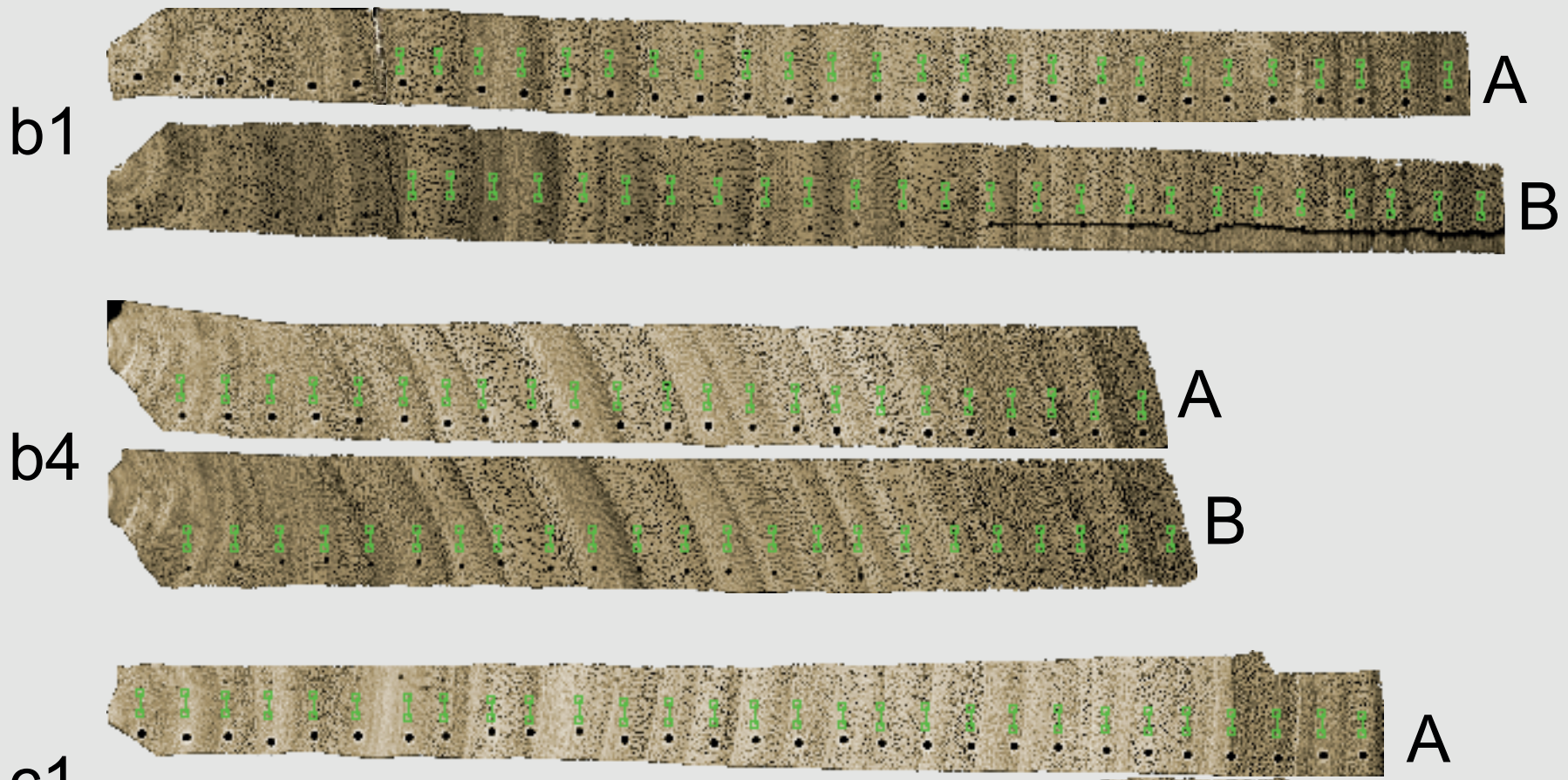

c1
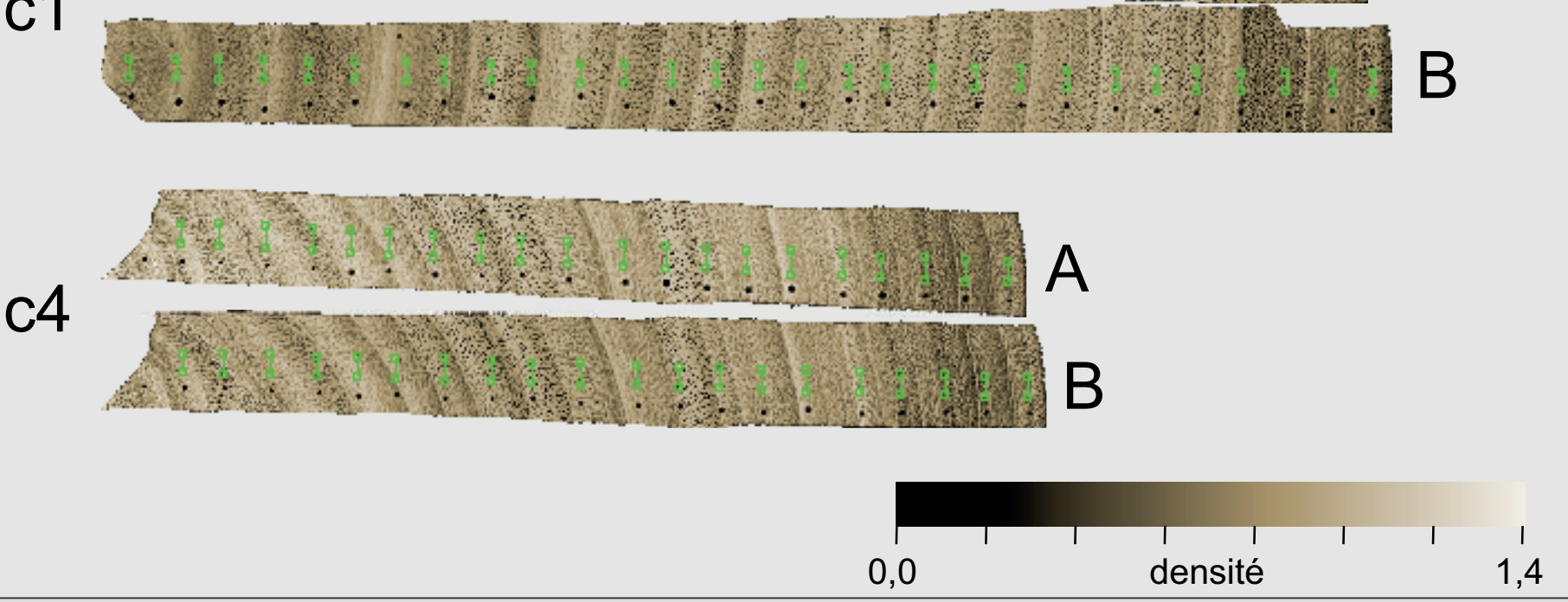

Figure 3.

Images calibrées en densité du bois des éprouvettes avant (A) et après (B) reconditionnement.

Les carrés verts représentent les points de contrôle utilisés pour synchroniser les deux profils. 


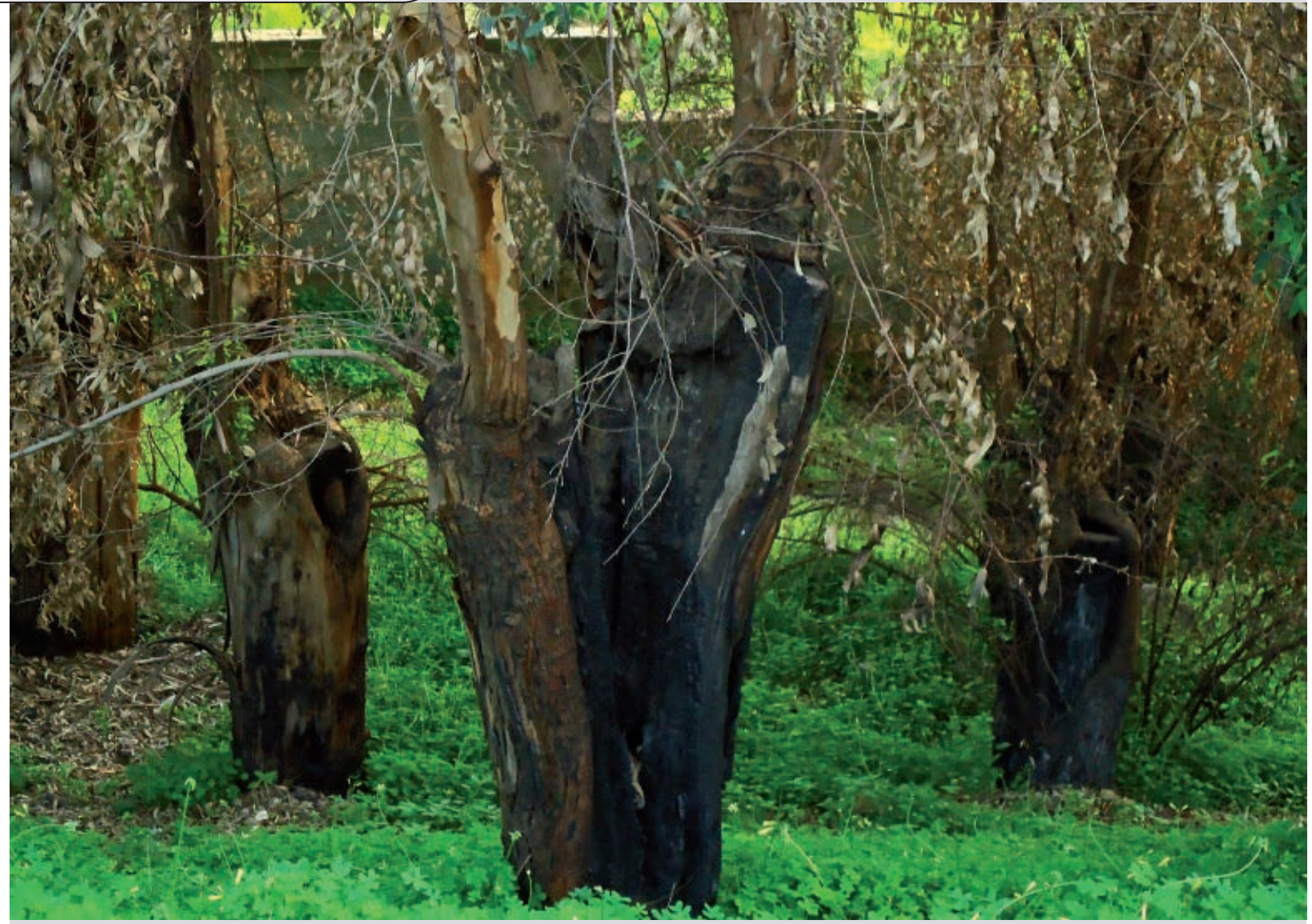

Photo 7.

Arbres d'Eucalyptus camaldulensis Dehn en bordure du chemin de fer ayant subi l'attaque d'insectes phoracantha, Phoracantha semipunctata, et psylle, Ctenarytaina eucalypti, (Boumerdès).

Photo M. Tazrout.

Le programme calcule pour chaque fenêtre 20 valeurs moyennes de densité, permettant ainsi de reconstruire un profil radial comprenant le même nombre de données avant et après reconditionnement. Chaque donnée des profils obtenus correspond donc à la densité moyenne d'une fenêtre haute de $2 \mathrm{~mm}$ dans la direction tangentielle et large d'un vingtième de la distance entre deux trous de perçage (soit environ 0,25 $\mathrm{mm}$ avant reconditionnement).

\section{Calcul des indicateurs du collapse}

Le reconditionnement d'une éprouvette se traduit par un gonflement d'autant plus important que l'éprouvette était collapsée. Le gain de volume relatif peut donc être considéré comme un indicateur global du collapse :

$\mathrm{ICV}=(\mathrm{Vr}-\mathrm{Vc}) / \mathrm{Vc}$

où Vc est le volume de l'éprouvette collapsée, avant reconditionnement, et $\mathrm{Vr}$ est le volume de l'éprouvette après reconditionnement.

Les volumes Vc et Vr sont calculés à partir des épaisseurs mesurées au pied à coulisse et des surfaces calculées sur les images radiographiques par le programme Cerd.

Le changement de volume se traduit par des variations de la densité locale, permettant donc le calcul en chaque point des profils d'un indicateur local du collapse par la perte de densité relative :
$I C d=(D c-D r) / D C$

où Dc est la densité du bois avant reconditionnement et $\mathrm{Dr}$ est la densité après reconditionnement.

Les deux indicateurs ICv et ICd doivent théoriquement rester proches de 0 en l'absence de collapse et augmenter avec la gravité du collapse. À la différence d'ICv, qui est un indicateur global, ICd présente l'avantage de pouvoir être calculé en tout point des profils de densité et de permettre donc d'analyser la variabilité radiale du collapse.

La différence d'humidité constatée entre les deux états, plus élevée de $0,5 \%$ après reconditionnement, devrait affecter légèrement les deux indicateurs en conduisant à surévaluer le gain de volume ${ }^{3}$ et sous-évaluer la perte de densité. Il convient de considérer néanmoins ici que les deux indicateurs traduisent les changements de propriétés générés par le collapse beaucoup plus que par le phénomène de retrait normal du bois au séchage.

L'analyse des résultats à ce stade montre que la synchronisation des profils avant et après reconditionnement est correcte, mais tout de même insuffisamment précise pour éviter des oscillations à haute fréquence de l'indicateur ICd, lequel atteint même localement des valeurs négatives. Pour mettre en relation le collapse avec la densité du bois, les trois profils ont subi un lissage (Dc, Dr et ICd) par moyenne mobile sur 10 valeurs, soit environ $2,5 \mathrm{~mm}$. 


\section{Résultats et discussion}

La densité moyenne par éprouvette varie entre 804 et $918 \mathrm{~kg} / \mathrm{m}^{3}$ avant reconditionnement et diminue de 8 à $16 \%$ après reconditionnement (tableau II). Le gain de volume (ICV) dû au reconditionnement est de $12,5 \%$ en moyenne contre $11,3 \%$ pour la perte de densité (ICd).

Les deux indicateurs donnent des résultats sensiblement identiques sur l'ensemble des éprouvettes, la différence s'expliquant probablement par les sources d'erreur mentionnées précédemment (écart d'humidité entre les mesures avant et après reconditionnement, sous-évaluation du volume des éprouvettes a1 et a4).

Le gonflement en volume concerne essentiellement la dimension tangentielle : celle-ci augmente de 7,8 \% en moyenne contre $2,1 \%$ pour la longueur (dimension radiale) et 2,2\% pour l'épaisseur (dimension longitudinale). Le rapport entre les gonflements tangentiel et radial $(3,7)$ est donc légèrement supérieur aux valeurs couramment rencontrées dans la littérature (1,5 à 3).

En moyenne par éprouvette (figure 4), les indicateurs de collapse sont d'autant plus faibles que la densité est élevée $(r=-0,95$ entre les moyennes par éprouvette de $D r$ et ICd, par exemple), ce qui est conforme à la littérature. L'arbre le moins dense (b) est ainsi celui qui présente les indicateurs de collapse les plus élevés.

L'effet hauteur mentionné dans la littérature est en revanche peu marqué et semble plutôt s'expliquer par l'effet de la densité : l'indicateur ICd est ainsi plus faible en bas de l'arbre (a) qui est le seul à présenter une densité plus élevée à $2 \mathrm{~m}$ qu'à $6,5 \mathrm{~m}$.

La mise en correspondance des images radiographiques avec les profils de densité (figure 5) confirme bien que les limites de cernes sont peu distinctes sur Eucalyptus camaldulensis Dehn (VALENZIANo, SCARAmuzzI, 1967).

Les vaisseaux les plus larges apparaissent nettement sous la forme de points noirs (à faible densité) mais les vaisseaux de plus faible diamètre ne sont pas visibles. Entre les zones de vaisseaux se distinguent de fines bandes tangentielles claires, ne contenant que des fibres, qui se traduisent sur les profils par des pics de densité.

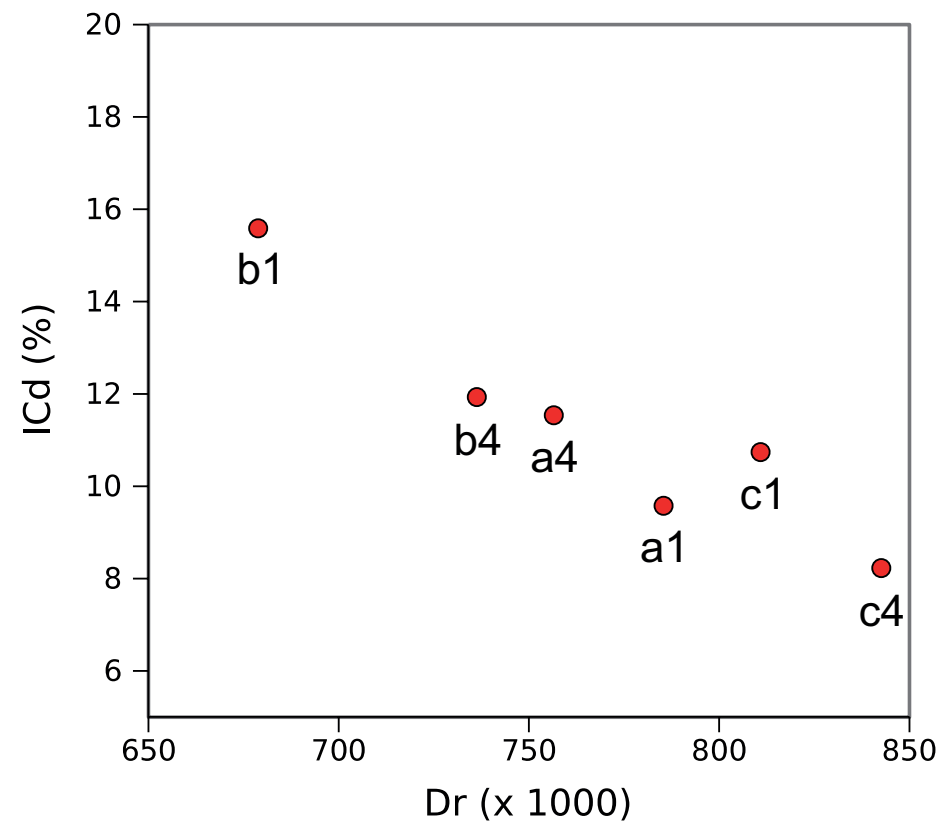

Figure 4.

Relation entre les moyennes par éprouvette de la perte de densité relative $(\mathrm{ICd})$ et de la densité après reconditionnement (Dr).

Également, la densité décroît brutalement d'environ $200 \mathrm{~kg} / \mathrm{m}^{3}$ dans la partie droite des profils. Cette chute de densité, que l'on retrouve sur toutes les éprouvettes à l'exception de a1, correspond probablement à la limite entre l'aubier et le duramen, plus riche en extractibles.

Les profils de densité avant et après reconditionnement sont très bien corrélés entre eux ( $r$ > 0,9 pour toutes les éprouvettes ; tableau II) mais la différence entre Dr et Dc n'est ni homogène, ni proportionnelle à la densité initiale, comme le montrent les irrégularités du profil de l'indicateur ICd.

\begin{abstract}
Tableau II.
Densités moyennes avant et après reconditionnement (Dc et Dr), indicateurs de collapse (ICd et ICv) et coefficients de corrélation entre les mesures densitométriques pour chaque éprouvette.
\end{abstract}

\begin{tabular}{|c|c|c|c|c|c|c|c|c|}
\hline Échantilon & Dc & Dr & $\mathrm{ICd}$ & $\mathrm{ICV}$ & Coeffic & ents de co & rélation & Nombre de mesures \\
\hline & $\left(\mathrm{kg} / \mathrm{m}^{3}\right)$ & $\left(\mathrm{kg} / \mathrm{m}^{3}\right)$ & (\%) & (\%) & $\mathrm{Dc} / \mathrm{Dr}$ & Dc / ICd & $\mathrm{Dr} / \mathrm{ICd}$ & \\
\hline a1 & 868 & 785 & 9,6 & 10,8 & $0,94^{\star \star \star}$ & $-0,04 \mathrm{NS}$ & $-0,38 * \star \star$ & 560 \\
\hline a4 & 855 & 757 & 11,5 & 10,1 & $0,92^{\star \star \star}$ & $0,38^{\star * *}$ & $-0,02 \mathrm{NS}$ & 560 \\
\hline b1 & 804 & 679 & 15,6 & 19,5 & $0,93^{\star * *}$ & $0,17 * \star$ & $-0,19 * \star$ & 480 \\
\hline b4 & 836 & 736 & 11,9 & 13,7 & $0,92^{\star \star \star}$ & $0,42^{* \star *}$ & $0,03 \mathrm{NS}$ & 340 \\
\hline c1 & 908 & 811 & 10,7 & 11,8 & $0,93^{* \star *}$ & $0,36^{\star * \star}$ & $0,02 \mathrm{NS}$ & 560 \\
\hline c4 & 918 & 843 & 8,2 & 8,9 & $0,96 * \star \star$ & $0,40 * \star \star$ & $0,13 \mathrm{NS}$ & 380 \\
\hline
\end{tabular}




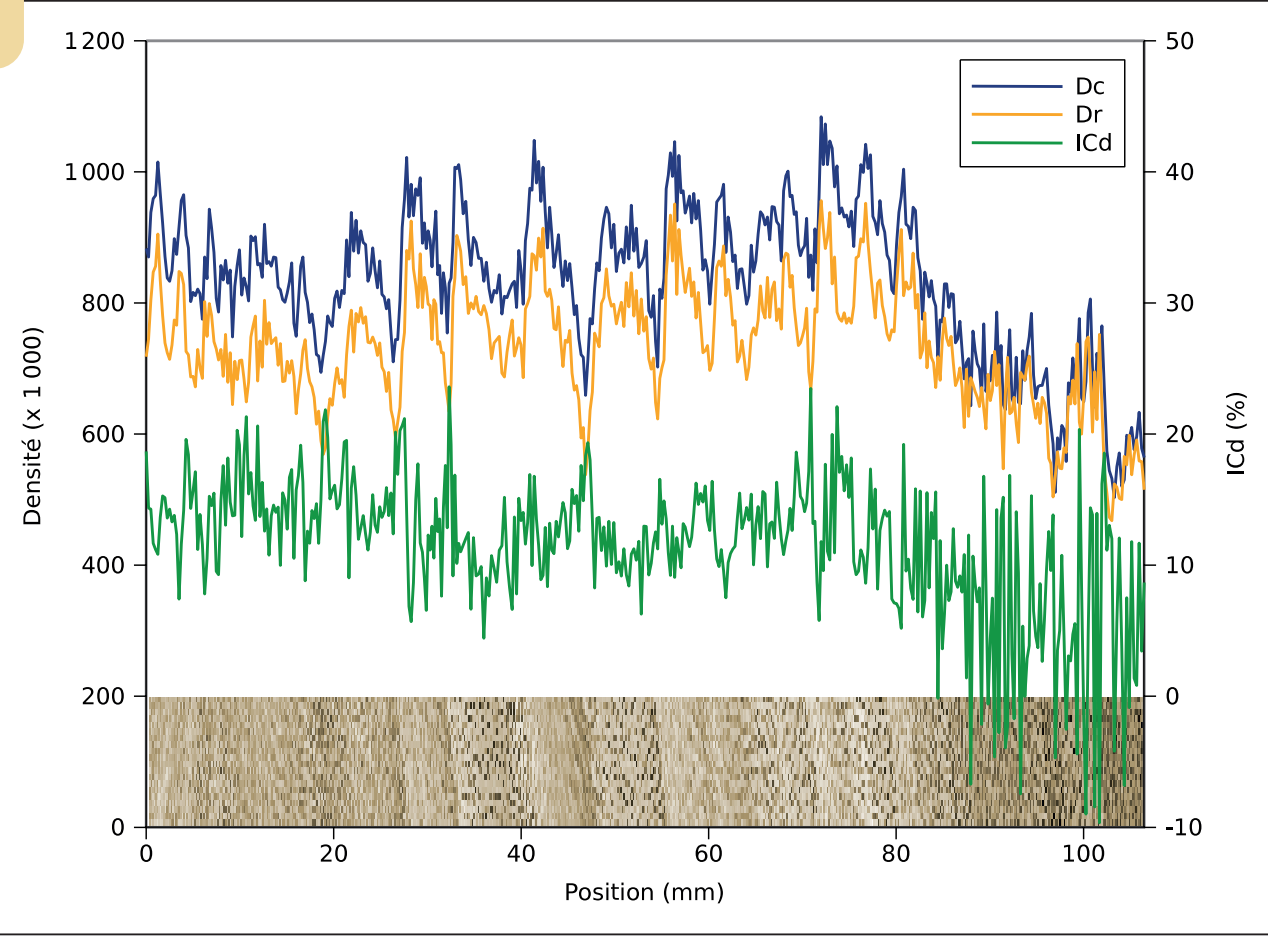

Figure 5.

Profils de la densité avant reconditionnement (Dc) et après reconditionnement (Dr) et de la perte de densité relative (ICd).

Éprouvette b4 ; données non lissées ; l'abscisse 0 correspond au point de mesure le plus proche de la moelle ; l'image en bas de la figure représente la carte de densité (Dc) de la zone de mesure.

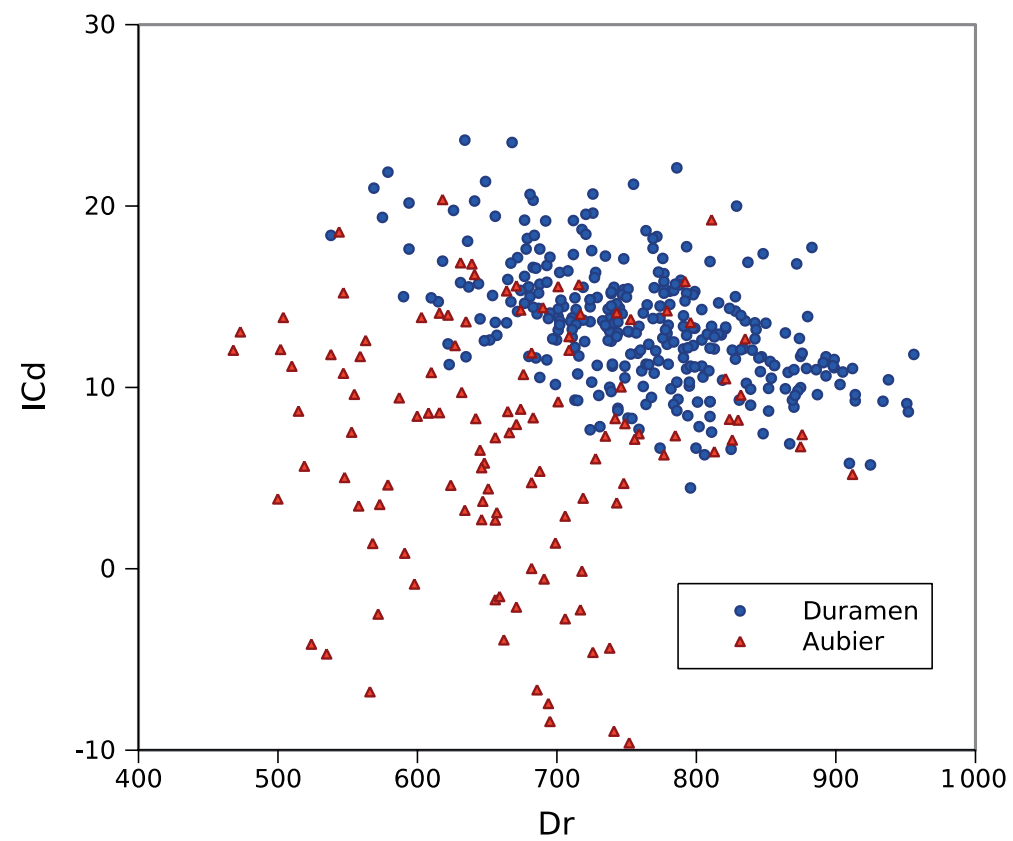

Figure 6.

Relation entre la perte de densité relative (ICd) et la densité après reconditionnement (Dr) dans l'aubier et le duramen.

Éprouvette b4 ; données non lissées ; la limite entre aubier et duramen a été fixée arbitrairement à $85 \mathrm{~mm}$ du début des profils.
Par construction, les coefficients de corrélation avec ICd sont en général positifs pour Dc et négatifs pour Dr (tableau II) mais il ne semble pas y avoir de relation claire entre la perte de densité relative et la densité. La figure 6 montre cependant, dans le cas de l'éprouvette b4, que la relation semble bien exister dans le duramen $(r=-0,52)$ mais ne se retrouve pas dans l'aubier $(r=0,03)$, où la perte de densité relative reste faible en moyenne malgré une densité sensiblement plus faible que dans le duramen. Le résultat est logique dans la mesure où l'écart de densité entre aubier et duramen s'explique probablement par une teneur différente en extractibles, plutôt que par des différences anatomiques qui pourraient avoir des effets sur la sensibilité au collapse.

Le lissage des profils (figure 7) a pour effet d'atténuer le bruit dû aux défauts de synchronisme entre les profils Dc et Dr. La décroissance marquée de l'indicateur ICd coïncidant avec la chute de densité apparaît alors clairement sur toutes les éprouvettes (à l'exception de a1), ce qui semble bien confirmer l'hypothèse que l'aubier serait peu affecté par le collapse.

Les éprouvettes de l'arbre (a) présentent toutefois une zone particulière (entre les abscisses 50 et $70 \mathrm{~mm}$ pour a1, 0 et $20 \mathrm{~mm}$ pour a4) dans laquelle ICd est anormalement faible. II pourrait s'agir aussi bien d'une zone non collapsée que d'un défaut de reconditionnement. Le profil de densité ne présente pas de particularité notable dans ces régions (si ce n'est peut-être l'absence de contraste entre bois initial et bois final). Une analyse anatomique de ces régions permettrait peutêtre de comprendre les raisons de ce comportement particulier.

Le lissage effectué sur les profils facilite aussi l'identification des limites de cernes dans certaines zones où apparaissent des ondulations marquées. 



Figure 7.

Profils lissés de la densité avant reconditionnement (Dc) et après reconditionnement (Dr)

et de la perte de densité relative (ICd). Moyennes mobiles sur 10 valeurs (environ 2,5 mm).

Celles-ci sont entrecoupées de plages de densité plus homogène - correspondant probablement à des cernes fins ou peu contrastés - dans lesquelles les limites de cernes ne sont pas discernables. Là où les limites de cernes sont apparentes, les variations de l'indicateur ICd sont clairement opposées aux profils de densité : ce sont les zones les moins denses qui présentent les pertes de densité les plus élevées. Ce résultat vient à l'appui des observations de BISSET et ELLWOOD (1951) concernant la plus grande sensibilité au collapse des cellules à parois fines du bois initial. 


\section{Conclusion}

La comparaison de profils densitométriques obtenus avant et après reconditionnement d'éprouvettes d'Eucalyptus camaldulensis Dehn collapsées, selon une méthodologie adaptée de Polge (1966) et Sesbou (1981), a permis de calculer un indicateur densitométrique de collapse et d'analyser ses variations radiales en relation avec la densité du bois.

Cette analyse a montré la pertinence de cet indicateur en permettant de retrouver plusieurs résultats connus de la littérature :

- l'indicateur de collapse est corrélé négativement à la densité moyenne des éprouvettes ;

- l'indicateur de collapse, comme la densité, est sensiblement plus faible dans l'aubier que dans le duramen;

- dans les zones où les limites de cernes sont apparentes, la plus forte sensibilité au collapse des cellules à paroi fine du bois initial se traduit par une évolution opposée des profils de l'indicateur et de la densité.

La procédure utilisée pour calculer cet indicateur de collapse est peu coûteuse expérimentalement et pourrait être appliquée sans difficultés à un large échantillonnage pour permettre l'étude approfondie des relations entre anatomie du bois et sensibilité au collapse.

Par ailleurs, la technique du reconditionnement mise en œuvre ici expérimentalement s'est avérée efficace pour récupérer une grande partie du collapse. Elle pourrait avantageusement s'appliquer en milieu industriel pour une meilleure valorisation du produit ligneux, en Algérie comme dans les autres pays dans lesquels les eucalyptus ont été introduits.

\section{Remerciements}

Les auteurs tiennent à remercier MM. Romain Rémond et Patrick Perré (Engref, Nancy, France) pour leurs conseils et leur assistance dans la réalisation de ces expériences.
ARMSTRONG L. D., 1983. Mechano-sorptive deformations in collapsible and non- collapsible species of wood. Journal of the Institute of Wood Science, 83: 206-211.

BARISKA M., 1975. Collapse phenomena in beechwood during $\mathrm{NH}_{3}$-impregnation. Wood Science and Technology, 9: 293-306.

BISSET I. J. W., ELLWOOD E. L., 1951. The relation of differential collapse and shrinkage to wood anatomy in Eucalyptus regnans F.v.M. and Eucalyptus gigantea Hook. F. Australian Journal of Applied Science, 2 (1): 175-183.

BLAKEMORE P. A., 2008. Optimisation of steam reconditioning for regrowth-ash and plantation growth-ash and plantation grown eucalypt specie. PhD Thesis, University of Sydney, Australie, $327 \mathrm{p}$.

BLAKEMORE P. A., NORTHWAY R., 2009. Review of, and recommendations for, research into preventing or ameliorating drying related internal and surface checking in commercially important hardwood species in south-eastern Australia. Final report received by FWPA in November, 2009. Reference Number: PNB047-0809.

BLAND D. E., 1971. The relation of lignin and polyphenol content of Tasmanian alpine ash (Eucalyptus delegatensis R. T. Bak.) to shrinkage and recovery. Wood Science and Technology, 5: 17-26.

CHAFE S. C., 1985. The distribution and interrelationship of collapse, volumetric shrinkage, moisture content and density in trees of Eucalyptus regnans F. Muell. Wood Science and Technology, 19: 329-345.

CHAFE S. C., 1986. Variation in moisture content, percent saturation and collapse following felling in trees of Eucalyptus regnans F. Muell. Australian Forest Research, 16: 175-184.

CHAFE S. C., ILIC J., 1992a. Shrinkage and collapse in thin sections and blocks of Tasmanian mountain ash regrowth. Part I: Shrinkage, specific gravity and fibre saturation point. Wood Science and Technology, 26: 115-129.

CHAFE S. C., ILIC J., 1992b. Shrinkage and collapse in thin sections and blocks of Tasmanian mountain ash regrowth. Part II: The R-ratio and changes in lumen volume. Wood Science and Technology, 26: 181-187.

CHAFE S. C., ILIC J., 1992c. Shrinkage and collapse in thin sections and blocks of Tasmanian mountain ash regrowth. Part III: Collapse. Shrinkage. Wood Science and Technology, 26: 343-351.

CHUDNOFF M., 1953. The effect of zinc chloride on some shrinkage properties of Eucalyptus camaldulensis wood. Ilanoth, 2: 5-16.

CHUDNOFF M., 1961. The physical and mechanical properties of Eucalyptus camaldulensis. Bulletin of the National Institute of Agricultural Bet. Dangan, $n^{\circ} 66$. 
CLARKE S. A., 1927. The Seasoning of Western Australian Hardwood with special reference to the structure, properties and air and kiln seasoning of Jarrah and Karri. Forests Department of Western Australia Bulletin, 40, 82 p.

CUNNINGHAM P., 1827. Two years in New South Wales. $2^{\text {nd }}$ ed. Londres, Royaume-Uni, Henry Colburn, vol. 1, 193 p.

ELLIOT C. S., 1923. Problems in the seasoning of hardwoods. Australian Forestry Journal, 6: 290-294.

ELLIOT C. S., 1930. Collapse of timber. A major cause of waste in the Australian timber industry. Journal, CSIR, 3: 204-211.

GREENHILL W. L., 1938. Collapse and its removal: some recent investigations with Eucalyptus Regnans. CSIR, Australia, Technical Paper, 24: 1-32.

HART C. A., 1970. Water filled lumens in steady-state flow in oak and poplar. Wood and Fiber, 1 (4): 308-318.

HAWLEY L. F., 1931. Wood liquid relations. U.S. Department of Agriculture Technical Bulletin, 248, 34 p.

ILIC J., 1999. Shrinkage-related degrade and its association with some physical properties in Eucalyptus regnans F. Muell. Wood Science and Technology, 33: 425-437.

KANAGAWA, 1977. Influence of cell collapse on the shrinkage process of wood. University of Nagoya, Japon.

KAUMAN W. G., 1958. The influence of drying stresses and anisotropie on collapse in Eucalyptus regnans. Melbourne, Australie, CSIRO, Division of Forest Products Technological Paper $\mathrm{n}^{\circ} 3$.

KAUMAN W. G., 1960a. Contributions to the theory of cell collapse in wood: investigations with Eucalyptus regnans. Australian Journal of Applied Science, 11 (1): 122-145.

KAUMAN W. G., 1960b. Collapse in some eucalypts after treatment in inorganic salt solutions. Forest Production Journal, 10 (9): 461-467.

MACKAY J. F. G., 1972. Recovery of collapse in Eucalyptus delegatensis by use of anhydrons ammonia and steam. Wood and Fiber, 4 (3): 126-129.

MOTHE F., DUCHANOIS G., ZANNIER B., LEBAN J.-M., 1998. Analyse microdensitométrique appliquée au bois : méthode de traitement des données utilisée à l'Inra-Erqb (programme Cerd). Annales des Sciences Forestières, 55 : 301-313.

PANKEVICIUS E. R., 1961. Influence of position in tree on recoverable collapse in wood. Forest Production Journal, 11 (3): 131-132.

PANKEVICIUS E. R., 1962. Collapse intensity for two eucalypts after treatment with hydrochloric acid and sodium chloride solutions. Forest Production Journal, 12 (1): 39-42. POLGE H., 1966. Établissement des courbes de variation de densité du bois par exploration densitométrique de radiographies d'échantillons prélevés à la tarière sur des arbres vivants. Applications dans les domaines technologique et physiologique. Thèse de doctorat, Université de Nancy-l, Champenoux, France, 215 p.
RUNKEL R. O. H., 1954. Studien über die Sorption der Holzfaser. Erste Mitteilung: Die Sorption der Holzfaser in morphologische-chemischer Betrachtung. Holz als Roh- und Werkstoff, 12 (6): 226-232.

SCHNIEWIND A. P., 1960. On the nature of drying stresses in wood. Holzforschung, 14 (6): 161-168.

SESBOU A., 1981. Étude de la variabilité génétique de la qualité du bois et du collapse chez Eucalyptus camaldulensis Dehn. Thèse de docteur-ingénieur, Université de NancyI, Faculté des sciences, France, 135-141.

STAMM A. J., LOUGHBOROUGH W. K., 1942. Variation in shrinkage and swelling of wood. American Society of Mechanical Engineers, 64: 379-386.

STILWELL S. T., STEVENS W. C., 1931. Elm, Beech and ash. The effect of high temperature steaming on their seasoned state. The Timber Trades Journal, December 12.

SUMMERS R. E., 1929. Reconditioning of collapsed hardwood, etc. Commonwealth of Australia, Munitions Supply Lab., Technical Notes, 28/1, 29/1 et 29/4.

THIERCELIN F., PERRIN J. R., 1972. Débit d'échantillons d'épaisseur rigoureusement uniforme à l'aide de sciesfraises jumelées. Annales des Sciences Forestières, 30 (2) : 295-299.

TIEMANN H. D., 1913. Eucalyptus Lumber. Hardwood record, Chicago, September 25 and October 10.

TIEMANN H. D., 1915. Principles of kiln drying. Lumber World Review, Janury 15 and September 25.

TIEMANN H. D., 1929. How to restore collapsed timber. The Lumber Worker, 5 (57): 37-44.

TIEMANN H. D., 1941. Collapse as shown by the microscope. Journal of Forestry, 39: 271-282.

TIEMANN H. D., 1948. Permeability of wood to air and liquids. Soap foam tests. Southern Lumberman, 177 (2223): 68.

VALENZIANO S., SCARAMUZZI G., 1967. Preliminary observations on the seasonal diameter growth of Eucalyptus camaldulensis and Eucalyptus viminalis. World symposium on man-made forest and their industrial importance. Rome, Italie, Fao, vol. 13 (1923-1935).

WU Y.-Q., HAYASHI K., LIU Y., CAI Y., SUGIMORI M., 2006. Relationships of anatomical characteristics versus shrinkage and collapse properties in plantation-grown eucalypt wood from China. Journal of Wood Science, 52 (3): 187-194. 